\title{
Jetzt neu: Masterstudienlehrgang „Radiological Technologies“ am DIW-MTA
} für MTRA

Das Deutsche Institut zur Weiterbildung für Technologen/-innen und Analytiker/-innen in der Medizin (DIW-MTA) bietet ab 2021 in Kooperation mit der Fachhochschule Gesundheit (fhg - Zentrum für Gesundheitsberufe Tirol $\mathrm{GmbH}$ ) in Innsbruck (Österreich) einen Masterlehrgang Radiologietechnologie am Standort Lüdenscheid (NRW) an, der zum Großteil online angeboten wird. Für MTRA besteht so die Möglichkeit, berufsbegleitend einen international anerkannten
Masterabschluss in Radiologietechnologie (MSc) zu erwerben.

Bewerben Sie sich jetzt für den neuen Masterstudienlehrgang „Radiological Technologies“, der ab September 2021 (Wintersemester 2021/22) startet! Der berufsbegleitende Masterstudienlehrgang richtet sich an alle MTRA, die nicht nur ihr Fachwissen, sondern auch ihre Managementfähigkeiten wissenschaftlich fundiert erweitern möchten. Die
Dauer beträgt 5 Semester, die Zulassung zum Studium ist auch ohne Abitur möglich (zusätzliche Prüfung erforderlich). Der Masterstudienlehrgang hat einen hohen OnlineAnteil, die Präsenzzeiten finden in Lüdenscheid/NRW statt. Alle weiteren Informationen rund um den Masterstudienlehrgang „Radiological Technologies“ finden Sie auf www.diw-mta.de. Gerne können Sie uns auch eine E-Mail mit Ihren Fragen an in fo@diw-mta.de schicken. 